\title{
Visual discrimination of abstract mirror-reflected three-dimensional objects at many orientations
}

\author{
LAWRENCE M. PARSONS \\ Massachusetts Institute of Technology, Cambridge, Massachusetts
}

\begin{abstract}
This study examined people's discrimination of identical and mirror-reflected pairs of abstract three-dimensional objects like those used by Shepard and Metzler (1971). In contrast to previous research, the axis of the orientation difference (OD) between a pair of objects was quite unconstrained by experimental design. Each stimulus pair differed in orientation by an angle of rotation up to $180^{\circ}$ about one of 13 disparate axes. The axis of OD was randomly ordered across trials. Subjects reported attempting to imagine one object at the orientation of the other and then comparing their shapes. Reaction time (RT) depended on the extent of OD, confirming earlier results. However, RT also depended on the axis or plane of OD between a stimulus pair. Slopes for RT$O D$ functions for trials with identical objects varied by a factor of 3 for different axes. The rank order of functions from steepest to slightest slope was: (1) axes not in any principal plane of the observer's visual reference frame, (2) frontal diagonal axes, (3) horizontal diagonal axes, (4) the line-of-sight axis, (5) midsagittal diagonal axes, (6) the vertical axis, and (7) the horizontal axis perpendicular to the line of sight. There was no difference in performance between subjects achieving high scores on pretests of spatial ability and those achieving moderate scores. The variation in slopes is likely due to variation in rate, path length, and/or initiation time of imagined reorientations. Various geometrical procedures could underlie the imagined spatial transformations used in the task. RT patterns are much more consistent with the class of procedures producing paths of relatively efficient length than the class of inefficient procedures, such as that using rotations through Euler angles.
\end{abstract}

When similar objects are at the same orientation, people can often readily discriminate differences in the composition and spatial arrangement of the objects' features. However, as the objects differ in orientation, the effort needed to discriminate between them increases. Searching for and comparing corresponding features of objects at different orientations can overburden spatial working memory (Parsons, in press). One very often finds it more efficient to imagine or physically produce rotation(s) of one object to the orientation of the other (e.g., Hinton \& Parsons, 1987; Shepard \& Metzler, 1971). The latter fact has been exploited to study both the internal representation of shape (e.g., Corballis, Zbrodoff, Shetzer, \& Butler, 1978; Hinton \& Parsons, 1981) and imagined spatial transformation (Just \& Carpenter, 1985; Metzler \& Shepard, 1974; Parsons, 1983a, 1983b, 1987b, 1987c, 1987d).

\section{Properties of Imagined Spatial Transformations}

The time required to imagine an object's reorientation often increases with the angle of orientation difference (e.g., Cooper \& Shepard, 1973; Metzler, 1973; Parsons, 1983b, 1987d). For those stimuli that have been examined

This research was supported by a grant from the A. P. Sloan Foundation Program in Cognitive Science to the MIT Center for Cognitive Science, and NRSA Fellowship F32 HD6605-02 from the National Institutes of Health. The author thanks Steve Pinker for useful comments on an earlier version of this manuscript. L. M. Parsons is with the Department of Brain and Cognitive Sciences, Massachusetts Institute of Technology, Cambridge, MA 02139. in detail (e.g., one's own body; Parsons, 1987b), the rate of imagined reorientation for different planes or axes can vary by a factor of at least 3 . In addition, the rate can vary by a factor of at least 2.5 for different directions within a plane of orientation difference (i.e., for clockwise and counterclockwise rotations about an axis; Parsons, 1987b). For other stimuli (e.g., those used by Shepard \& Metzler, 1971), the rate may not vary with the plane or direction of reorientation. Finally, the rate of imagined reorientation is apparently influenced by properties of the imagined object. Rates can vary by more than an order of magnitude depending on the object's complexity or familiarity (cf. results in Cooper, 1975; Kaushall \& Parsons, 1981; Parsons, 1987b; Shepard \& Hurwitz, 1984; Shepard \& Metzler, 1971).

These and related results (e.g, Cooper, 1976; Pinker, 1980) are taken to imply that the imagined spatial transformations produce an approximately continuous series of intermediate internal representations of a shape that correspond to its intermediate physical orientations. In addition, these results are thought to imply that objects are internally represented in three dimensions, rather than in two dimensions of projected three-dimensional information (as in a literally "pictorial" representation).

\section{Limits of Previous Imagined \\ Spatial Transformation Studies}

In most previous research, one or the other of two planes (or axes) of rotation were most efficient to correct for the difference in orientation between a standard and 
comparison object. Orientation differences (ODs) were in one or two planes (picture or depth), and trials were often blocked by the plane of OD.

Such experimental designs fail to reflect an important aspect of human spatial transformations. The perceptual, imaginal, or motor systems are capable of interpolating, recognizing, representing, or effecting the efficient displacement of an object from one orientation to (apparently) any other, with little deliberation. When a threedimensional object is imagined moving from one orientation to another, it seems to arrive there by a systematically executed and direct path. It does not move about randomly until happening upon its goal. We show similar economy in our actions. In addition, we can perceive whether an object not under our control moves efficiently to a desired orientation. We can also experience (or interpolate) efficient apparent motion between pairs of orientations of an object.

Assuming that we can imagine, perceive, and produce any arbitrary path, an unresolved issue is the nature of the procedures and economies that allow us to select from among the infinitely many paths an object can traverse. To understand this issue, researchers have begun to study imagined spatial transformations, attempting to develop conditions under which imagined paths can be compared to those produced by models based on different kinds of computational and geometrical procedure (Just \& Carpenter, 1985; Parsons, 1983b, 1987b, 1987c, 1987d). The experiment reported here is part of this effort and is an initial examination of this problem in the paradigm used by Shepard and Metzler (1971). The early studies by Metzler and Shepard (1974) investigated some aspects of this problem by examining the visual discrimination and imagined reorientation of abstract three-dimensional objects (Figure 1). They found that reaction time (RT) was longer if the OD could be in one of two planes rather than always being in a single plane. This suggests that the time to initiate a reorientation (e.g., to find or plan a path) is not constant and is influenced by constraints of the experimental design. Their results also suggest that with $O D$ in only one of these two planes, people imagine rotation through the shorter of the two possible angles about an axis.

\section{DISCRIMINATION OF OBJECT PAIRS AT MANY DISPARATE ORIENTATION DIFFERENCES}

In Shepard and Metzler's (1971) studies, observers were of high spatial ability and objects were presented at orientations differing in two principal planes of the observer's visual frame of reference. Subjects in the present experiment also discriminated between identical and mirrorreflected pairs of shapes like those used by Shepard and Metzler. However, the stimuli were presented in 13 disparate and randomly ordered planes of OD. The object pairs were separated by an OD about one of the three principal axes of the observer's reference frame; about a diagonal axis lying within the frontal, midsagittal, or
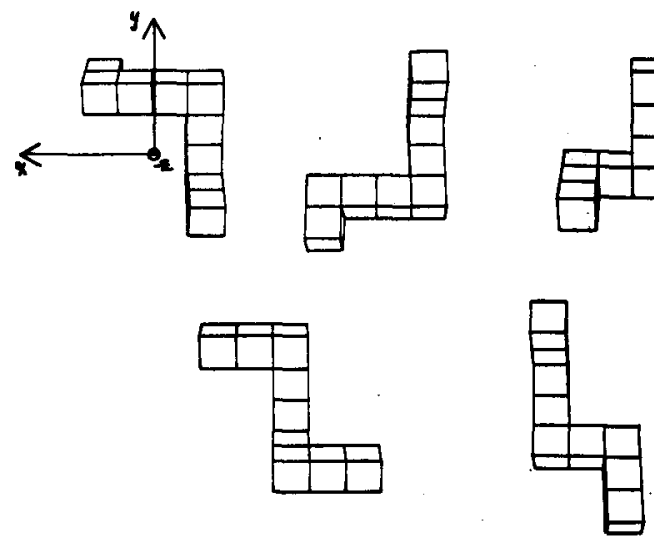

Figure 1. Five stimuli at the "standard" orientation used to create the set of orientations from which the stimulus pairs were selected (see Method section). Reading left to right, Objects 1, 2, and 3 are in the top row, and Objects 4 and 5 are in the bottom row. The $x$-, $y$-, and $z$-axes imposed on Object 1 are like those imposed on each object in order to construct the set of stimuli used here. The axes of rotation referred to are described with respect to the frame of reference composed of these three axes. (The negative $z$-axis is perpendicular to this page and pointed toward the observer.)

horizontal plane of that reference frame; or about a "skew" axis not lying in any of the principal planes. Ten subjects participating in the experiment were of high spatial ability and 10 were of moderate spatial ability (see Table 4).

The purpose of the study reported here was to provide a baseline collection of discrimination RT-OD functions on which further, more critical, empirical and theoretical work can be grounded. It examined discrimination functions at a representative subset of possible ODs. This affords some initial examinations of subjects' abilities and preferences for selecting planes or axes for imagined reorientations. These data can potentially discriminate among classes of spatial transformation procedures that differ with respect to the general efficiency of their reorientation paths. "Simulation"' studies (such as those of Cooper, 1975; Metzler, 1973; Parsons, 1987b, 1987c) and spatial reasoning studies can be used later to examine in more detail the properties of imagined spatial transformations.

\section{Three Factors That May Affect RT'}

First, the rate of imagined reorientation may depend on the plane or direction of OD. Differences in rates for different reorientations may reflect properties of the information processing that underlies imagined spatial transformations. More computation may be required for reorientations that cause an object's parts to "disappear" or "reappear" from the observer's perspective. Rotation about some axes may be ecologically common and more rapid than rotation about others. (The results of Kaushall \& Parsons, 1981, suggest that the effect of familiarity and practice on implied rotation rate can be profound.) Parsons (1987b) studied the imagined rotation of one's body about various axes and found that the slope of the RT func- 
tion for the line-of-sight axis was six times that for the vertical axis.

Second, the time to initiate the spatial transformation of an object from one orientation to another may vary with the plane or direction of reorientation. The amount of computation required to determine or plan a path can vary for different spatial transformation procedures, depending on various properties of the OD between objects (Parsons, 1987a). It may require less time to find or plan an imagined path when the OD is in a principal plane of the object or of the observer's visual frame of reference. The path or axis of rotation may be more "obvious" when $O D$ is in such principal planes (on salience of the vertical axis, see Clark, 1973; Corballis \& Roldan, 1975; Rock, 1973).

The third possible influence on the observed RTorientation functions is that the total degrees of effective imagined rotation may depend on the plane or direction of OD. There are an infinite number of paths for passing an object from one orientation to another, and a path can be produced by more than one spatial transformation procedure (cf. Parsons, 1987a). Although there are many different possible spatial transformation procedures, for purposes of illustration I will briefly discuss only three basic approaches (see Appendix; see Parsons, 1987a, for more details): (1) Rotations by dimensions: a sequence of rotations about a different axis (e.g., a principal axis of the object or environment) for each dimension by which they differ in orientation (e.g., intrinsic dimensions such as front-back, left-right, and top-bottom). ${ }^{2}$ (2) Spin precession: rotation about an instantaneously changing axis produced by simultaneous rotations about two orthogonal axes (e.g., a principal axis of the object and an axis of the environment). (3) Shortest path: rotations about an axis (unique for each OD) to simultaneously correct for all differences in orientation while absolutely minimizing the degrees of rotation. Procedures 2 and 3 are examples of the class of procedures that in general produce paths of relatively efficient length; Procedure 1 is an example of the class of relatively inefficient procedures. Each of these procedures (or variants thereof) uses the same angle of rotation and path when the orientation difference is due to rotation about a single principal axis, either of the object or of the observer's visual reference frame or environment. Most previous work could not discriminate among different reorientation procedures because only ODs in such principal planes were used. (The exceptions are Cooper \& Shepard's [1975] study of hands [but this issue did not arise in their analysis-see Parsons, 1987c]; Just \& Carpenter, 1985; Parsons, 1983b, 1987b.)

\section{Possible Experimental Outcomes}

One possibility is that all the discrimination functions are identical, or nearly so, in linear shape, slope, and intercept. This would be consistent with several previous conclusions (Attneave, 1972; Metzler \& Shepard, 1974; Shepard, 1975, 1982, 1984). ${ }^{3}$ Another possible outcome is that discrimination functions for different planes of $O D$ vary in slope and intercept. This may occur if the rates, initiation times, and/or path length of imagined spatial transformations differ with the plane of $O D$. Observed RT-OD functions can be compared with the predicted degrees of rotation (see Appendix A) for the spatial transformation procedures described earlier.

\section{Method}

\section{Subjects}

Twenty right-handed MIT undergraduates, who had not been in any related experiments, participated for $\$ 5 / \mathrm{h}$. All subjects completed two timed paper-and-pencil pretests of the ability to imagine spatial transformations of objects (Thurstone's Cube Comparison and Card Rotation tests; Ekstrom, French, Harman, \& Dermen, 1976). Ten subjects achieved high scores on both tests, correctly answering an average of $88.75 \%$ of the items (individuals ranged from $95 \%$ to $85 \% ; S D=3.47$ ), with an average error rate of .0275 . Ten others achieved moderate scores on the tests, completing an average of $69.1 \%$ of the items correctly (individuals ranged from $80 \%$ to $62 \% ; S D=5.51$ ), with an average error rate of .0326

\section{Stimuli}

Black-and-white photographic slides of pairs of line drawings of abstract three-dimensional objects (Figure 1) were illuminated by a Mast slide projector (with attached tachistoscopic shutter). Five computer-generated, orthographically projected objects and their five mirror reflections were presented simultaneously in pairs in an arrangement like that in Figure 2. The objects subtended between $4^{\circ}$ and $5^{\circ}$ of visual angle. The pairs consisted of two views of the same object or an object and its mirror reflection.

Stimuli were presented at orientations sampled from a larger set of orientations created by the following method. First, an axis of rotation (see Figure 3) was passed through the center of mass of an object at its standard orientation (Figure 1), and the object was rotated from $30^{\circ}$ to $330^{\circ}$ in $30^{\circ}$ intervals. The Cartesian reference frame used to describe the axes of rotation was oriented with respect to each object (as in Figure 1). From the resulting set of orientations about each axis, pairs of orientations of an object were selected (1) that differed in relative orientations by $30^{\circ}, 60^{\circ}, 90^{\circ}, 120^{\circ}$, $150^{\circ}$, or $180^{\circ} ;(2)$ in which as little as possible of the object's shape was occluded or distorted by pictorial depiction; and (3) in which, despite the object's relative OD, its orientations varied as much as possible over all its pairs, with respect to the fixed Cartesian
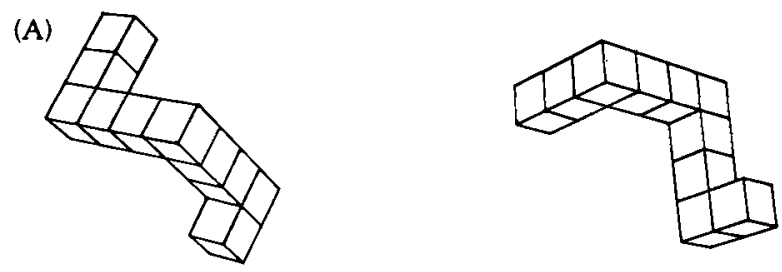

(B)
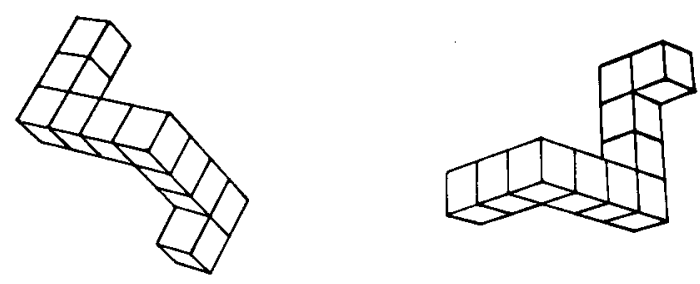

Figure 2. Appearance of stimuli. (A) An identical pair, and (B) a mirror-reflected pair. Each stimulus of the pair was encircled by a dark background. The center of mass of each object was located at the center of the circular surround. 

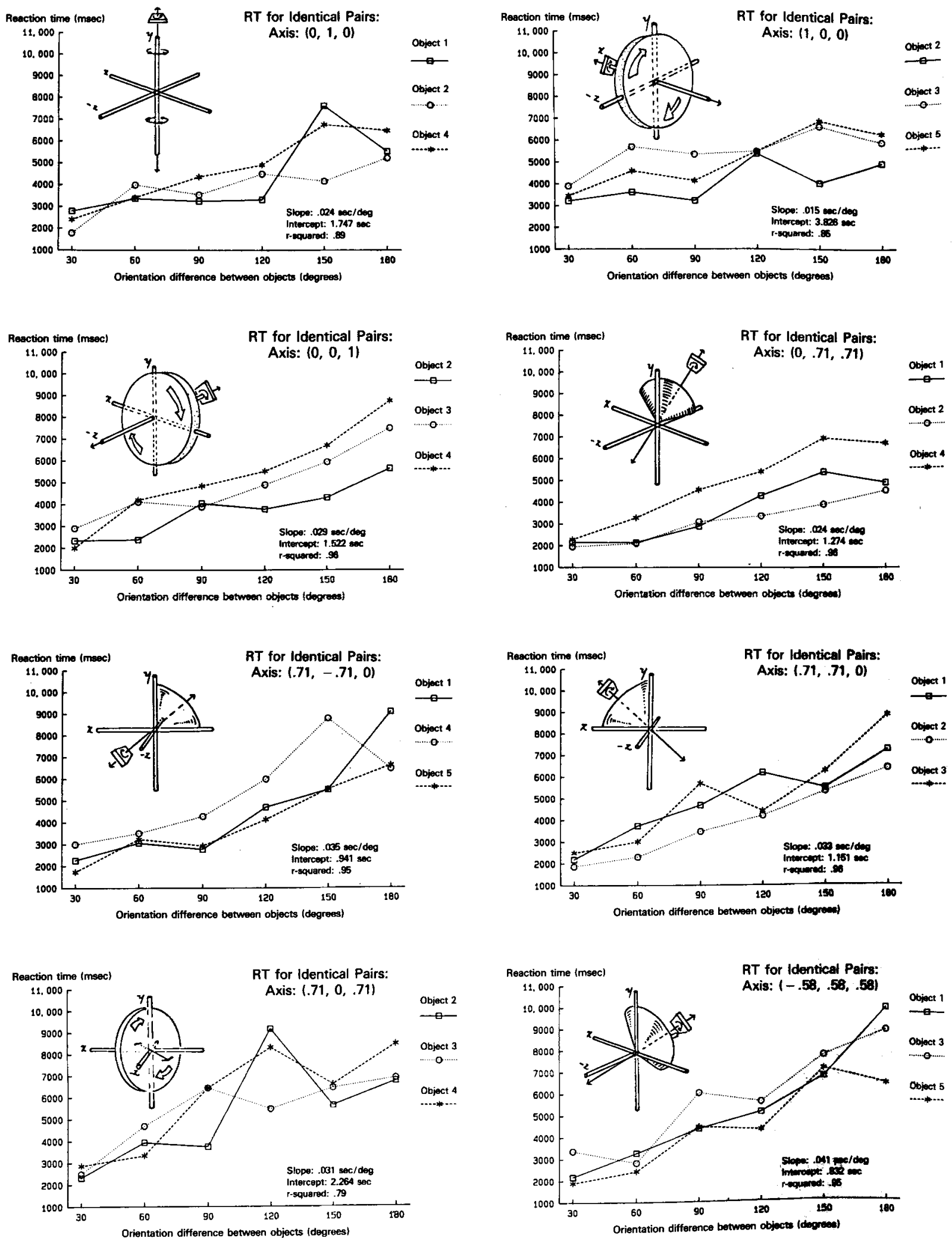

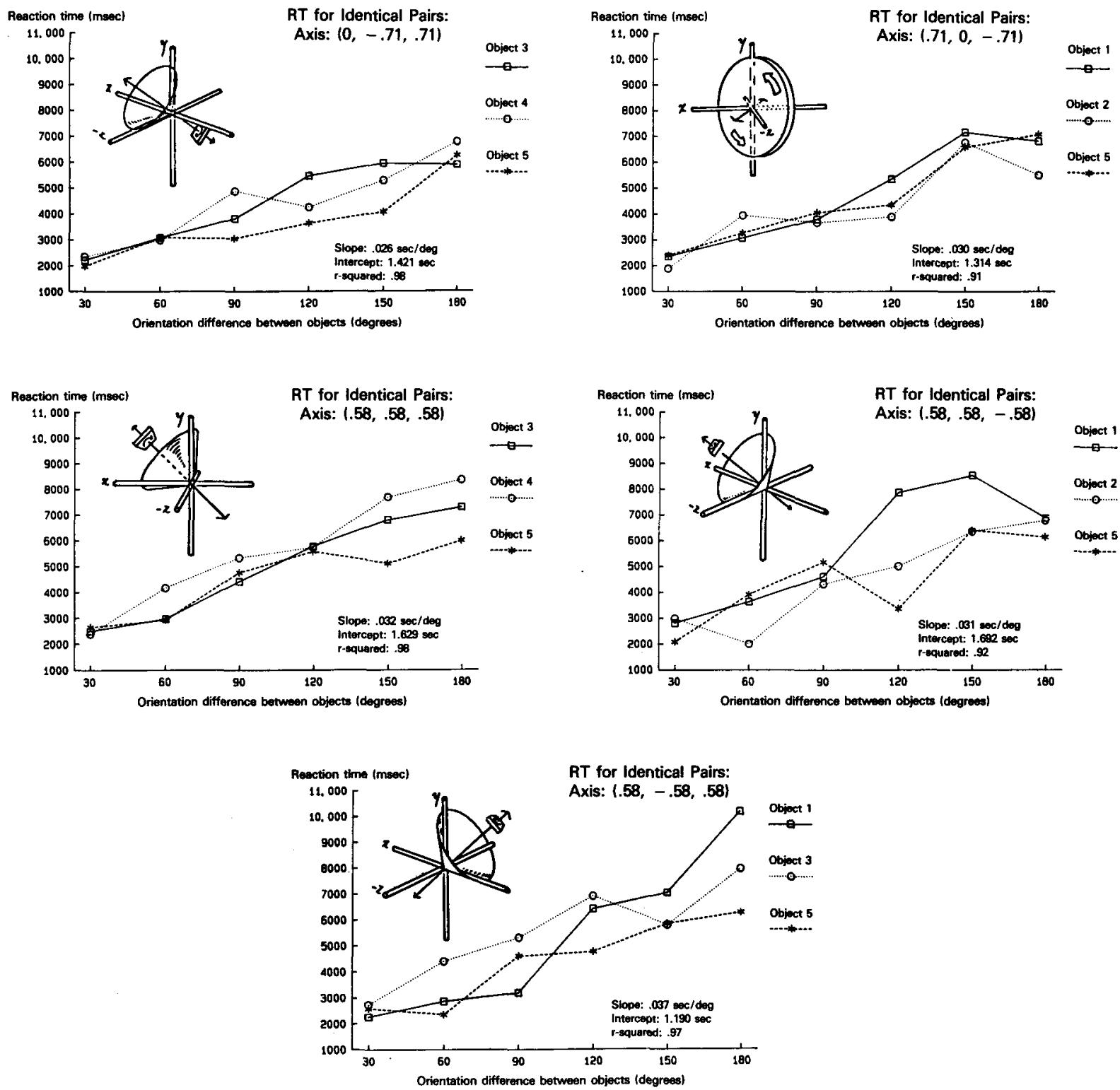

Figure 3. RT as a function of extent and axis of orientation difference between pairs of identical objects. The axis of rotation used to create the orientation difference between stimuli is depicted in the npper left-hand corner of the corresponding RT plot. Subjects' line of sight on the stimuli was always down the depicted negative $z$-axis toward the origin (Figure 1). For the purpose of clearly depicting the orientation of an object about an axis of rotation, the $x$ - and $z$-axes are in some cases shifted with respect to the plane of this page. In the lower right-hand corner is the slope and intercept of best-fit linear regression equation of RT on OD collapsing across the three objects, and the amount of variance accounted for by this regression.

frame of the environment. For pairs of stimuli with no difference in orientation, a single view of each object (and its mirror image) was selected from the total set of stimuli on the basis of how well the object's surfaces were depicted. For three of the five objects, the views used for the $0^{\circ}$ OD pairs were also placed at the various orientations in the picture plane to produce stimuli for the $(0,0,1)$ axis. Three objects were presented at orientations about each axis of rotation (Table 1).

Objects in a mirror-reflected pair were oriented as in Figure 2: two of the corresponding segments of both objects were identically oriented, and the remaining segments of one object were oriented in directions opposite to those of corresponding segments of the other object. Trials on which the objects were identical always used the five objects in Figure 1 (not their mirror images).

\section{Design}

Each subject completed three replications of 478 trials resulting from the presentation of a mirror-reflected and an identical pair of three objects at six orientation differences $\left(30^{\circ}, 60^{\circ}, 90^{\circ}, 120^{\circ}\right.$, $150^{\circ}$, and $180^{\circ}$ ) about 13 axes, and two pairs (mirror and identical) of five objects at an identical orientation. The first replication was practice. Within each replication, trials were performed in a random order unique for each subject.

\section{Procedure}

Subjects sat before a screen with their index fingers on a twokey microswitch, and pressed a left button for a pair of mirrorreflected objects and a right button for a pair of identically shaped objects. They were to respond as rapidly and accurately as possi- 
Table 1

Object and Axis of Rotation Creating Orientation Difference

\begin{tabular}{llllll}
\hline \multicolumn{1}{c}{$\begin{array}{c}\text { Axis of } \\
\text { Orientation Difference }\end{array}$} & $\mathrm{1}$ & $\mathbf{2}$ & $\mathbf{3}$ & $\mathbf{4}$ & $\mathrm{5}$ \\
\hline \multicolumn{1}{c}{$(1,0,0)$} & $\mathrm{X}$ & $\mathrm{X}$ & $\mathrm{X}$ & & $\mathrm{X}$ \\
$(0,1,0)$ & $\mathrm{X}$ & & $\mathrm{X}$ & \\
$(0,0,1)$ & & $\mathrm{X}$ & $\mathrm{X}$ & $\mathrm{X}$ & \\
$(.71,0, .71)$ & $\mathrm{X}$ & $\mathrm{X}$ & $\mathrm{X}$ & \\
$(.71, .71,0)$ & $\mathrm{X}$ & $\mathrm{X}$ & $\mathrm{X}$ & & \\
$(.71,-.71,0)$ & $\mathrm{X}$ & & & $\mathrm{X}$ & $\mathrm{X}$ \\
$(0, .71, .71)$ & $\mathrm{X}$ & $\mathrm{X}$ & & $\mathrm{X}$ & \\
$(0,-.71, .71)$ & & & $\mathrm{X}$ & $\mathrm{X}$ & $\mathrm{X}$ \\
$(.71,0,-.71)$ & $\mathrm{X}$ & $\mathrm{X}$ & & & $\mathrm{X}$ \\
$(.58, .58, .58)$ & & & $\mathrm{X}$ & $\mathrm{X}$ & $\mathrm{X}$ \\
$(.58, .58,-.58)$ & $\mathrm{X}$ & $\mathrm{X}$ & & & $\mathrm{X}$ \\
$(-.58, .58, .58)$ & $\mathrm{X}$ & & $\mathrm{X}$ & & $\mathrm{X}$ \\
$(.58,-.58, .58)$ & $\mathrm{X}$ & & $\mathrm{X}$ & & $\mathrm{X}$ \\
\hline
\end{tabular}

ble and were not to make head or hand movements. A PDP-11/03 microcomputer controlled the slide projectors and recorded RT and correctness of response. Subjects completed the experiment in eight 1-h sessions conducted on alternate days. Trials on which errors were made were repeated later in a block until performed correctly.

\section{Results}

\section{Error Rates}

Error rate was less than .03 on average (.02 for high spatial ability group and .04 for moderate spatial ability group), and was correlated with RT $[r=.50, F(1,237)$ $=79.45, p<.0001]$ for each of the three standard objects (or their mirror images) about each OD about each axis.

\section{Analyses of RT Data}

Analyses used RTs for correct responses only. An analysis of variance (ANOVA) of RTs for each object, using axis, orientation, and identity, showed no reliable differences between subjects in the high and moderate spatial ability groups, so other analyses of RTs collapsed across moderate and high spatial ability groups.

RT was linearly related to the OD between object pairs, but also depended both on the axis used to create the difference in orientation and on the relationship between axis and object (Figure 3).

An identical pattern of reliable effects was observed in a separate ANOVA (Table 2) of RTs for each object (with 6 ODs, 2 identities, and 8 or 7 axes) and in an ANOVA collapsing across objects (with 6 ODs, 13 axes, and 2 identities). RT was reliably affected by the axis and extent of $O D$. The effect of OD on RT was affected by axis. RT was different for identical and mirror-reflected pairs. This difference between mirror and identical pairs depended separately on the extent and axis of OD. Finally, the difference in RT between identical and mirror pairs depended on the interaction of axis and extent of OD.

In an ANOVA of RTs for identical pairs, a model adding the ANOVA factors involving axis of OD to the usual model of mental rotation (which uses only extent of OD) accounts for $20 \%$ more of the total sums of squares accounted for than the model using only extent of orientation difference (.54 compared to .45 ).
RT-OD Functions for Trials With Identical Objects

The slope of the best-fit linear RT-OD functions depended on the axis of rotation used to create the OD. Figure 3 shows the best-fit linear regression of OD on RT for pairs of identical objects, for each axis collapsed across objects.

Although RT-OD functions depended on both the axis and the object, there was no reliable difference between slopes of the best-fit linear regression for each kind of axis for different objects (two-tailed $t$ test, $p>.05$ ). Collapsing across objects, the slopes for individual axes range from $.041 \mathrm{sec} / \mathrm{deg}$ for axis $(-.58, .58, .58)$ to $.015 \mathrm{sec} / \mathrm{deg}$ for axis $(1,0,0)$. In rank order, the average slopes for each set of related axes are as follows. Steepest slopes were observed for "skew" axes $(.0353 \mathrm{sec} / \mathrm{deg})$, followed by diagonal axes within the frontal plane (.034), horizontal diagonal axes (.305), line-of-sight axis (.029), diagonal axes within the midsagittal plane $(.025)$, vertical axis (.024), and the horizontal axis perpendicular to the line of sight (.015).

An ANOVA of slopes of best-fit linear regression equations of RT on OD, for identical trials for each subject, shows a reliable effect of axis on the slope $[F(12,228)=$ $8.89, p<.001]$. Many of the slopes of RT-OD functions are reliably different (by Newman-Keuls tests, $p<.05$ ).

\section{Predictions of Different \\ Spatial Transformation Procedures}

Linear regressions were used to compare RT with the predicted degrees of rotation for passing one object to the orientation of the other using each of three spatial transformation procedures. (This test used the degrees of rotation shown in Table 3 and mean RTs for trials with identical objects, for each axis and each OD.) The spin-precession and shortest-path procedures both fit RTs very well $\left[r^{2}=.85, F(1,76)=406.75, p<.0001\right.$, and $r^{2}=.85,[F(1,76)=397.44, p<.0001$, respectively $]$. The rotations-by-dimensions procedure (using a path pass-

Table 2

Analysis of Variance $F$ Values

\begin{tabular}{|c|c|c|c|c|c|c|}
\hline \multirow[b]{2}{*}{ Factor } & \multicolumn{5}{|c|}{ Object } & \multirow{2}{*}{$\begin{array}{l}\text { Collapsing } \\
\text { Across All } \\
\text { Five Objects }\end{array}$} \\
\hline & 1 & 2 & 3 & 4 & 5 & \\
\hline Axis of OD & 13.63 & 41.03 & 16.28 & 29.95 & 12.68 & 32.51 \\
\hline Extent of OD & 44.99 & 46.40 & 34.18 & 57.87 & 57.34 & 88.68 \\
\hline Axis $\times$ Extent & 4.61 & 10.17 & 5.84 & 5.12 & 7.20 & 8.12 \\
\hline Identity & 34.58 & 30.45 & 39.71 & 95.47 & 23.13 & 59.65 \\
\hline Identity $\times$ Axis & 7.21 & 15.30 & 6.55 & 8.20 & 4.99 & 11.79 \\
\hline Identity $\times$ Extent & 57.72 & 35.95 & 53.17 & 75.32 & 12.47 & 157.82 \\
\hline Identity $x$ & & & & & & \\
\hline Extent $\times$ Axis & 2.28 & 5.74 & 3.59 & 2.02 & 3.72 & 5.10 \\
\hline
\end{tabular}

Note-All $F$ values are reliable to at least $p<.001$. The degrees of freedom are, for objects $1-3$ and 5 ; axis $(7,133)$; extent $(5,95)$; axis and extent $(35,665)$; identity $(1,19)$; identity and axis $(7,133)$; identity and extent $(5,95)$; identity, extent, and axis $(35,665)$. For ANOVA for object 4 : axis $(6,114)$, extent $(5,95)$, axis and extent $(30,570)$, identity $(1,19)$, identity and axis $(6,114)$, identity and extent $(5,95)$, and identity, axis, and extent $(30,570)$. For ANOVA over all objects: axis $(12,228)$, extent $(5,95)$, axis and extent $(60,1140)$, identity $(1,19)$, identity and axis $(12,228)$, identity and extent $(5,95)$, and identity, extent, and axis $(60,1140)$. 
Table 3

Degrees of Rotation Between Pairs of Objects Using Each of Three Spatial Transformation Procedures

Orientation Differences

\begin{tabular}{|c|c|c|c|c|c|c|}
\hline \multirow[b]{2}{*}{ Axis } & \multicolumn{6}{|c|}{ Urientation Dimerences } \\
\hline & $30^{\circ}$ & $60^{\circ}$ & $90^{\circ}$ & $120^{\circ}$ & $150^{\circ}$ & $180^{\circ}$ \\
\hline \multicolumn{7}{|c|}{ Rotations by Dimensions } \\
\hline $\begin{array}{l}(1,0,0) \\
(0,1,0) \\
(0,0,1) \\
(.71, .71,0) \\
(0, .71, .71) \\
(.71,0, .71) \\
(0,-.71, .71) \\
(.71,0,-.71) \\
(.71,-.71,0) \\
(.58, .58, .58) \\
(.58, .58,-.58) \\
(.58,-.58, .58) \\
(-.58, .58, .58)\end{array}$ & $\begin{array}{r}210^{\circ} \\
30^{\circ} \\
210^{\circ} \\
42^{\circ} \\
158^{\circ} \\
120^{\circ} \\
201^{\circ} \\
120^{\circ} \\
64^{\circ} \\
114^{\circ} \\
114^{\circ} \\
114^{\circ} \\
114^{\circ}\end{array}$ & $\begin{array}{r}240^{\circ} \\
60^{\circ} \\
240^{\circ} \\
85^{\circ} \\
132^{\circ} \\
150^{\circ} \\
221^{\circ} \\
150^{\circ} \\
130^{\circ} \\
138^{\circ} \\
138^{\circ} \\
138^{\circ} \\
138^{\circ}\end{array}$ & $\begin{array}{r}270^{\circ} \\
90^{\circ} \\
270^{\circ} \\
130^{\circ} \\
130^{\circ} \\
180^{\circ} \\
240^{\circ} \\
180^{\circ} \\
201^{\circ} \\
161^{\circ} \\
161^{\circ} \\
161^{\circ} \\
161^{\circ}\end{array}$ & $\begin{array}{l}300^{\circ} \\
120^{\circ} \\
300^{\circ} \\
177^{\circ} \\
177^{\circ} \\
210^{\circ} \\
255^{\circ} \\
210^{\circ} \\
278^{\circ} \\
180^{\circ} \\
180^{\circ} \\
180^{\circ} \\
180^{\circ}\end{array}$ & $\begin{array}{l}330^{\circ} \\
150^{\circ} \\
330^{\circ} \\
224^{\circ} \\
224^{\circ} \\
240^{\circ} \\
266^{\circ} \\
240^{\circ} \\
307^{\circ} \\
234^{\circ} \\
234^{\circ} \\
274^{\circ} \\
234^{\circ}\end{array}$ & $\begin{array}{l}360^{\circ} \\
180^{\circ} \\
360^{\circ} \\
270^{\circ} \\
270^{\circ} \\
270^{\circ} \\
270^{\circ} \\
270^{\circ} \\
270^{\circ} \\
290^{\circ} \\
290^{\circ} \\
290^{\circ} \\
290^{\circ}\end{array}$ \\
\hline \multicolumn{7}{|c|}{ Spin Precession } \\
\hline $\begin{array}{l}(1,0,0) \\
(0,1,0) \\
(0,0,1) \\
(.71, .71,0) \\
(0, .71, .71) \\
(.71,0, .71) \\
(0,-.71, .71) \\
(.71,0,-.71) \\
(.71,-.71,0) \\
(.58, .58, .58) \\
(.58, .58,-.58) \\
(.58,-.58, .58) \\
(-.58, .58, .58)\end{array}$ & $\begin{array}{l}30^{\circ} \\
30^{\circ} \\
30^{\circ} \\
30^{\circ} \\
30^{\circ} \\
30^{\circ} \\
30^{\circ} \\
30^{\circ} \\
30^{\circ} \\
30^{\circ} \\
30^{\circ} \\
30^{\circ} \\
30^{\circ}\end{array}$ & $\begin{array}{l}60^{\circ} \\
60^{\circ} \\
60^{\circ} \\
61^{\circ} \\
61^{\circ} \\
60^{\circ} \\
61^{\circ} \\
60^{\circ} \\
61^{\circ} \\
61^{\circ} \\
61^{\circ} \\
61^{\circ} \\
61^{\circ}\end{array}$ & $\begin{array}{l}90^{\circ} \\
90^{\circ} \\
90^{\circ} \\
93^{\circ} \\
93^{\circ} \\
90^{\circ} \\
93^{\circ} \\
90^{\circ} \\
93^{\circ} \\
93^{\circ} \\
93^{\circ} \\
93^{\circ} \\
93^{\circ}\end{array}$ & $\begin{array}{l}120^{\circ} \\
120^{\circ} \\
120^{\circ} \\
127^{\circ} \\
127^{\circ} \\
120^{\circ} \\
127^{\circ} \\
120^{\circ} \\
127^{\circ} \\
127^{\circ} \\
127^{\circ} \\
127^{\circ} \\
127^{\circ}\end{array}$ & $\begin{array}{l}150^{\circ} \\
150^{\circ} \\
150^{\circ} \\
163^{\circ} \\
163^{\circ} \\
150^{\circ} \\
163^{\circ} \\
150^{\circ} \\
163^{\circ} \\
163^{\circ} \\
163^{\circ} \\
163^{\circ} \\
163^{\circ}\end{array}$ & $\begin{array}{l}180^{\circ} \\
180^{\circ} \\
180^{\circ} \\
201^{\circ} \\
201^{\circ} \\
180^{\circ} \\
201^{\circ} \\
180^{\circ} \\
201^{\circ} \\
211^{\circ} \\
211^{\circ} \\
211^{\circ} \\
211^{\circ}\end{array}$ \\
\hline
\end{tabular}

Shortest Path

For all axes: $\quad 30^{\circ} \quad 60^{\circ} \quad \begin{array}{lllll} & 90^{\circ} & 120^{\circ} & 150^{\circ} & 180^{\circ}\end{array}$

*This procedure rotates an object through Euler angles (see text).

ing through Euler angles) fits less well $\left[r^{2}=.49, F(1,76)\right.$ $=74.58, p<.0001]$. The fit to the data is only slightly improved if a hybrid spatial transformation procedure is assumed in which the shortest-path procedure is used when the OD is in a principal plane of the observer's visual reference frame, and the rotations-by-dimensions procedure using Euler angles is used for other ODs $\left[r^{2}=.53\right.$, $F(1,76)=89.41, p<.0001]$.

The spin-precession and shortest-path procedures account for $73 \%$ more of the variance than does the rotations-by-dimensions procedure. Both observed slopes of RT-OD functions for various axes and the observed relationships among the set of overall means for each axis deviate from those predicted by the rotations-bydimensions procedure (using Euler angles).

\section{Trials With Pairs of Mirror-Reflected Objects}

Overall RT for trials with pairs of identical objects was $1,611 \mathrm{msec}$, and that for trials with pairs of mirrorreflected objects was 4,932 msec. The RT-OD function for trials with mirror-reflected objects had a slighter slope and higher intercept than those of trials with identical objects (the slopes were, respectively, .00518 and $.03013 \mathrm{sec} / \mathrm{deg}$; the intercepts were 5.136 and $1.583 \mathrm{sec}$ ). However, because objects with different shapes cannot be placed at identical orientations, it is unclear what is a valid plot of RTs. No further analysis was made of RTs on the trials with mirror-reflected objects.

\section{Subjects' Introspections}

Subjects reported attempting to imagine one object at the orientation of the other. Three subjects reported occasionally inferring shape by noticing planes of symmetry in an object pair and not imagining one object at the orientation of the other.

\section{Discussion}

\section{Discrimination RT Depends on the Axis of OD Between Members of a Pair of Objects}

These results confirm that the time to visually discriminate mirror-reflected and identical pairs of threedimensional objects varies with the extent of the objects' difference in orientation (e.g., Shepard \& Metzler, 1971). However, these results also indicate that the time to make this discrimination depends on the plane in which the objects differ in orientation. There appears to be no performance difference between subjects of high and moderate spatial ability.

These findings in some respects confirm, and in others differ from, the results of Shepard and Metzler's (1971) study. However, the differences do not seem to be inadvertently caused by differences in the experimental designs used. As shown in Table 4, the experimental designs are similar with respect to exposure to stimuli, practice at the discrimination task, and overall sample size. Accordingly, there are comparable introspections and overall means. There are also comparable slopes and intercepts for the two planes of OD that the two experiments have in common.

Only 3 of the 20 subjects reported occasionally using a strategy of first detecting symmetrical relations between the directions in which ends of segments of objects point and then making inferences as to the relation between the shapes of the two objects. There were no differences between these 3 subjects' RT-OD functions and those of the other subjects. Overall, the RT function characteristic of the use of this configurational strategy (i.e., a quadratic function with longest RTs at $90^{\circ}$ of orientation difference) was seldom observed. Apparently, then, the use of this strategy was not a major influence on RT-OD functions.

\section{Variation in Slopes of RT-OD Functions for Different Axes of OD}

There were reliable differences in slopes of the discrimination RT-OD functions for different axes or planes of orientation difference. For the most part, similar slopes were observed for functions of axes that have comparable spatial relationships to the principal planes of the observer's visual frame of reference. This suggests that the slopes were influenced more by the ODs created by these related axes than by (incidental) properties of the shapes, views, and relations between principal axes of the object and axes of rotation that were sampled. 
Table 4

Comparison of Shepard and Metzler's (1971) Experiment and the Present Experiment

\begin{tabular}{|c|c|c|}
\hline & Shepard \& Metzler (1971) & Present Experiment \\
\hline Subjects & 8 high spatial ability & $\begin{array}{l}10 \text { high spatial ability } \\
10 \text { moderate spatial ability }\end{array}$ \\
\hline Stimuli & \multicolumn{2}{|c|}{ Same 5 objects and mirror reflections } \\
\hline Object orientations & $\begin{array}{l}20^{\circ} \text { intervals about } 2 \text { principal } \\
\text { axes of observer's visual frame } \\
\text { of reference }\end{array}$ & $\begin{array}{l}30^{\circ} \text { intervals about } 3 \text { principal axes } \\
\text { of observer's visual reference frame } \\
\text { and } 10 \text { "arbitrary" axes (Figure } 3 \text { ) }\end{array}$ \\
\hline Number of trials & (40 practice) 1,600 test & (478 practice) 956 test \\
\hline Replications (for each subject) & 2 & 3 \\
\hline $\begin{array}{l}\text { Number of trials with each object } \\
\text { (and its mirror) for each subject }\end{array}$ & 328 & 318 \\
\hline $\begin{array}{l}\text { Number of trials with each axis } \\
\text { for each subject }\end{array}$ & $\begin{array}{l}\qquad 720 \\
\text { (sometimes blocked by axis } \\
\text { or plane of OD) }\end{array}$ & $\begin{array}{l}\text { (axis or plane of } \mathrm{OD} \\
\text { in random order) }\end{array}$ \\
\hline $\begin{array}{l}\text { Number of trials with an object at } \\
\text { an orientation about an axis } \\
\text { for a subject }\end{array}$ & 4 & $\begin{array}{c}1,2,3, \text { or } 4 \\
\text { (modal frequency }=1)\end{array}$ \\
\hline $\begin{array}{l}\text { Overall sample size } \\
\text { (Number of trials, across subjects, } \\
\text { with an object [and its mirror } \\
\text { image] at an orientation difference } \\
\text { about a particular axis) }\end{array}$ & 64 & 60 \\
\hline
\end{tabular}

The steepest slopes were observed for skew axes (.0353 $\mathrm{sec} / \mathrm{deg}$ on average; $.032, .031, .037$, and .041 individually), followed by diagonal axes within the frontal (picture) plane (.034 on average; .033 and .035 separately), horizontal diagonal axes (.0305 on average; .031 and .030 separately), line-of-sight axis (.029), diagonal axes within the midsagittal plane (.025 on average; .024 and .026 separately), vertical axis (.024), and the horizontal axis perpendicular to the line of sight $(.015)$. Thus, the slopes for principal axes of an observer's visual frame were slighter than those for most of the other axes. The slopes for skew axes were $56 \%$ greater than those for principal axes. The RT-OD function with the slightest slopes was produced when the axis of OD was horizontal and perpendicular to the observer's line of sight $(1,0,0)$. The steepest function was observed when the axis of OD was skewed upward, away from, and rightward of the observer (axis $[-.58, .58, .58]$ in Figure 3 ).

The slopes of RT-OD functions for individual axes varied by a factor of about 3 . This range is comparable to the range of variation for the other stimulus examined in detail in a discrimination task (a left-right judgment of the outstretched arm of a human body; Parsons, 1987b). The same planes of OD were used in the present study and in Parsons (1987b). However, the slopes and intercepts of the functions for the various planes of OD for discriminating the objects in the present study are uncorrelated with those observed for left-right judgment of the outstretched arm of a human body. ${ }^{4}$ Contrary to results of earlier studies (Cooper \& Podgorny, 1976; Cooper \& Shepard, 1975; Shepard, 1975), this difference in results for different kinds of stimuli is consistent with other re- cent results suggesting that properties of the object being imagined can strongly influence characteristics of imagined spatial transformation (see Parsons, 1987c, 1987d).

\section{Properties of Imagined Spatial \\ Transformations Used}

It is likely that the variations in RT-OD functions for different planes of OD are due to variation in the imagined spatial transformations used. In particular, this variation in functions is probably due to variation in the rate, initiation time, and/or path of imagined spatial transformations. If so, the following conclusions can be drawn.

No evidence that people use shortest-path rotations whose rates and initiation times are constant for different ODs. These data do not confirm the hypothesis that observers imagine one object at the orientation of the other by using a minimum-angle path with a rate and initiation time identical for different planes of $O D$. Thus, the prediction most consistent with the framework of Shepard and his co-workers (Cooper \& Shepard, 1973; Metzler \& Shepard, 1974; Shepard, 1975, 1982, 1984) is not confirmed.

Use of spatial transformations of efficient length. Assuming that rates and initiation times of imagined reorientations for different planes of OD vary little, the data suggest that subjects spontaneously use relatively efficient spatial transformation procedures. RTs are consistent with spatial transformation procedures producing paths approximately as efficient in length as those produced by the spinprecession or shortest-path procedures. These results demonstrate that at least one other spatial transformation procedure (i.e., spin-precession) fits a large and diverse 
set of data as well as the shortest path procedure which is generally (implicitly or explicitly) assumed to be used (see introductory remarks).

The RTs are much less consistent with use of a relatively inefficient procedure that produces paths like those produced by the rotations-by-dimensions procedure (using Euler angles). They are also much less consistent with the use of a spatial transformation procedure of shortestpath rotations for ODs about one of the principal axes of the observer's visual reference frame, and rotations-bydimensions reorientations (using Euler angles) for all other ODs.

These conclusions apply to both high and moderate spatial ability subjects, who showed no differences in performance. It is possible that the difference in spatial ability between groups here was not great enough to replicate the finding that high spatial ability subjects imagine more efficient spatial transformations than do subjects of low spatial ability (Just \& Carpenter, 1985).

The results here are consistent with those of a study by Parsons (1987b) of imagined spatial transformations of one's body. Those studies involved left-right judgment of the outstretched arm of one's misoriented body and the imagined reorientation of the body via a path of one's choice or via an experimenter-specified shortest path. The pattern of results suggested that for some planes of $O D$, subjects spontaneously used procedures that produced paths of quite efficient length (e.g., spin precession or shortest path). However, there was evidence against the hypothesis that people spontaneously use the shortest paths between any pair of orientations (Shepard, 1982, 1984). Subjects had difficulty learning the shortest paths for orientations about axes that were not in a principal plane of the body, and their performance reflected this. (This observation is consistent with analyses of the computational requirements of a shortest-path procedure [Parsons, 1987a] and with the author's own unpublished studies of spatial reasoning that suggest that people do not ordinarily know or use shortest paths between different orientations of an object if the spatial difference is not in one of the principal planes of the object or the observer's visual reference frame.)

Inferences about the rates of imagined reorientation. Even though it is apparent that people use procedures producing paths of efficient length, a relatively steep slope for a particular RT-OD function cannot be interpreted in general to imply that there is a slow rate of imagined reorientation in that plane of OD. There are many possible procedures that could produce reorientation paths approximately as efficient as those produced by the spinprecession and shortest-path procedures (Parsons, 1987a). Even if the effective degrees of rotation for two reorientation procedures are experimentally indistinguishable, the exact paths can differ. If it is assumed that people use either the spin-precession or shortest-path procedure, then we can examine the special-case ODs when the two procedures produce exactly the same paths (in the plane of $\mathrm{OD}$ ).
In those cases, the slope is $.015 \mathrm{sec} / \mathrm{deg}$ for the horizontal axis perpendicular to the line of sight, $.024 \mathrm{sec} / \mathrm{deg}$ for the vertical axis, $.029 \mathrm{sec} / \mathrm{deg}$ for the line-of-sight axis, and $.0305 \mathrm{sec} / \mathrm{deg}$ for the two horizontal diagonal axes. If it is further assumed that these slopes indicate the rate of reorientation in these planes of $O D$, then the results can be examined with respect to various other results and hypotheses.

Assuming, then, that in the special cases just described only one path is imagined and that it is always in the plane of OD, the following three points can be made:

1. The difference between the implied rotation rates for the vertical and line-of-sight axes is consistent with, although much smaller than, that in Parsons's (1987b) study of the imagined rotation of one's body through experimenter-specified shortest paths. The slope for the vertical axis in that study was six times that for the lineof-sight axis (.0065 compared to $.0010 \mathrm{sec} / \mathrm{deg}$ ). Rotation about the vertical axis is probably the most common of all rotations in our ecology, so the more rapid rate of imagined rotation may result from extraexperimental familiarity.

2. The fact that the rotation rate for most of the specialcase ODs or axes is not obviously slower than that for the line-of-sight axis (picture plane ODs) is consistent with Shepard and Metzler's (1971) results and conclusions (among others). They interpreted their data to indicate that RT was a function of the angle or rotation in three dimensions, not two, as in a retinal projection. This is consistent with the hypothesis that spatial and shape information is internally represented in three dimensions.

3. It is unclear why, under these assumptions, the rate of rotation about the horizontal axis perpendicular to the line of sight is half that of the line-of-sight and horizontal diagonal axes. This implies that the information processing underlying rotation about the former axis is performed more readily or quickly. This may relate to the fact that this kind of rotation does not alter the orientation of the "sides" of an object with respect to the observer's left and right, and this may cause the spatial transformation to be predictable or redundant.

\section{Conclusion}

As described earlier, there are a large number of possible geometrical methods on which unconstrained and/or natural spatial transformations could be based. This fact presents difficult-although not insurmountable-experimental problems for those studying the human use of spatial transformations. To precisely confirm the interpretations reported here, further work must examine the variation of reorientation rate and initiation time with different planes of OD. Such work should also discriminate among alternative spatial transformation procedures. This could be done by appropriate sampling of ODs and by appropriate variations in the relations among the object-centered principal axes, set of axes of OD, and the principal axes of the observer's visual frame of reference. 


\section{REFERENCES}

ATtNeave, F. (1972). Representation of physical space. In A. W. Melton \& E. Martin (Eds.), Coding processes in human memory (pp. 283306). Washington, DC: Winston.

ClARK, H. H. (1973). Space, time, semantics, and the child. In T. E. More (Ed.), Cognitive development and the acquisition of language. New York: Academic Press.

CoOPER, L. A. (1975). Mental rotation of random two-dimensional shapes. Cognitive Psychology, 7, 20-43.

COOPER, L. A. (1976). Demonstration of a mental analog of an external rotation. Perception \& Psychophysics, 19, 296-302.

CoOper, L. A., \& Podgorny, P. (1976). Mental transformations and visual comparison processes: Effects of complexity and similarity. Journal of Experimental Psychology: Human Perception \& Performance, 2, 503-514.

CoOper, L. A., \& SHEPard, R. N. (1973). Chronometric studies of the rotation of mental images. In W. G. Chase (Ed.), Visual information processing (pp. 75-176). New York: Academic Press.

COOPER, L. A., \& ShePARD, R. N. (1975). Mental transformations in the identification of left and right hands. Journal of Experimental Psychology: Human Perception \& Performance, 1, 48-56.

Corballis, M. C., \& Roldan, C. E. (1975). Detection of symmetry as a function of angular orientation. Journal of Experimental Psychology: Human Perception \& Performance, 1, 221-230.

Corballis, M. C., Zbrodoff, J., Shetzer, L. I., \&utler, P. B. (1978). Decisions about identity and orientation of rotated letters and digits. Memory \& Cognition, 6, 98-107.

Ekstrom, R. B., French, J. W., Harman, H. H., \& Dermen, D. (1976). Manual for kit of factor-referenced cognitive tests. Princeton, NJ: Educational Testing Service.

Goldstein, H. (1950). Classical mechanics. Reading, MA: AddisonWesley.

Hinton, G. E., \& Parsons, L. M. (1981). Frames of reference and mental imagery. In A. D. Baddeley \& J. Long (Eds.), Attention and performance (Vol. 9, pp. 261-277). Hillsdale, NJ: Erlbaum.

Hinton, G. E., \& PARsons, L. M. (1987). Scene-based and viewercentered representations for comparing shapes. Manuscript submitted for publication.

Just, M. A. \& Carpenter, P. A. (1985). Cognitive coordinate systems: Accounts of mental rotation and individual differences in spatial ability. Psychological Review, 92, 137-172.

Kaushall, P., \& Parsons, L. M. (1981). Optical information and practice in the discrimination of 3-D mirror-reflected objects. Perception, $10,545-562$.

METZLeR, J. (1973). Cognitive analogues of the rotation of threedimensional objects. Unpublished doctoral dissertation, Stanford University.

METZleR, J., \& SHEPARD, R. N. (1974). Transformational studies of the internal representation of three-dimensional objects. In R. Solso (Ed.), Theories in cognitive psychology: The Loyola Symposium (pp. 174-201). Hillsdale, NJ: Erlbaum.

PARSONS, L. M. (1983a). Imagined spatial transformations in the discrimination of left and right parts of the body. Proceedings of the Fifth Annual Conference of the Cognitive Science Society, Rochester, NY.

Parsons, L. M. (1983b). Mental rotation paths in the discrimination of left and right parts of the body. Unpublished doctoral dissertation, University of California, San Diego.

PARsons, L. M. (1987a). Evaluation and use of spatial transformation procedures in apparent motion and imagination. Manuscript submitted for publication.

PARsons, L. M. (1987b). Imagined spatial transformation of one's body. Journal of Experimental Psychology: General, 116, 172-191.

PArsons, L. M. (1987c). Imagined spatial transformation of one's hands and feet. Cognitive Psychology, 19, 178-241.

PARSONS, L. M. (1987d). Spatial transformations used in imagination, perception, and action. In L. Vaina (Ed.), Matters of intelligence (pp. 139-176). Dordrecht, The Netherlands: Reidel.

PARSONS, L. M. (in press). Serial search and comparison of pairs of objects. Memory \& Cognition.
PinKER, S. (1980). Mental imagery and the third dimension. Journal of Experimental Psychology: General, 109, 254-371.

Rock, I. (1973). Orientation and form. New York: Academic Press. SHEPARD, R. N. (1975). Form, formation and transformation of internal representation. In $\mathbf{R}$. Solso (Ed.), Information processing and cognition: The Loyola Symposium (pp. 87-117). Hillsdale, NJ: Erlbaum.

SHEPARD, R. N. (1982). Perceptual and analogical bases of cognition. In J. Mehler, M. Garrett, \& E. Walker (Eds.), Perspectives in mental representation (pp. 49-67). Hillsdale, NJ: Erlbaum.

SHEPARD, R. N. (1984). Ecological constraints on internal representation: Resonant kinematics of perceiving, imagining, and dreaming. Psychological Review, 91, 417-447.

SHEPARD, R. N., \& CoOPER, L. A. (1982). Mental images and their transformations. Cambridge, MA: MIT Press/Bradford Books.

SHEPARD, R. N., \& HuRwitZ, S. (1984). Spatial cognition, mental rotation, and interpretation of maps. Cognition, 18, 161-193.

SHEPARD, R. N., METZLER, J. (1971). Mental rotation of threedimensional objects. Science, 191, 952-954.

\section{NOTES}

1. A fourth factor that could influence the RT-orientation functions here is related to the comparison of shape required to discriminate between objects' shapes. People could search for a plane of symmetry between a pair of objects. This could allow them to infer the relationship of the two objects' shape without imagining a spatial transformation (Kaushall \& Parsons, 1981, p. 562). However, it turned out there was apparently little use of this strategy in Experiment 1 (see Discussion section), so it was not an important factor here.

2. Rather than examine all of the variants of the rotations-bydimensions procedure, I will focus on only one variant. This variant is moderately inefficient with respect to the total degrees of rotation and is therefore representative of the class of such procedures. This procedure rotates the object through Euler angles (Goldstein, 1950). These rotations are about the object's intrinsic principal axes. In addition, I will also consider a spatial transformation procedure that is a hybrid of the shortest path and rotations-by-dimensions procedures. In this hybrid, the shortest path is used when the orientation difference is in a principal plane of the observer's visuai reference frame; and the rotations-by-dimensions procedure using Euler angles is used for other ODs. This kind of model might be proposed if it were thought that people can use the shortest path reorientation only when it is "obvious" to them, as when the axis of OD is in a principal plane of their visual reference frame. Otherwise, they rely on other procedures.

3. These conclusions are as follows. First, that subjects use minimumangle reorientations to correct for any difference in orientation of two objects. Second, that the rate of imagined reorientation is approximately independent of the direction of rotation. Such a uniform outcome requires that the initiation time of imagined spatial transformations be constant across planes of orientation difference. This uniform outcome is also predicted if (1) subjects use a spin-precession procedure (described earlier) and (2) the rates and initiation times of imagined reorientation are constant across planes. In some cases of orientation difference here, the total degrees of rotation for the spin-precession procedure are somewhat greater than those for the shortest path. However, because of the underlying variability of RTs in this paradigm, it is impossible to distinguish performance with the two procedures. Furthermore, uniform functions would be strong evidence against a procedure such as rotations-by-dimensions. It wbuld be a very unlikely coincidence for the differences in rates and initiation times to exactly compensate for (or mask) the differences in the length of path predicted by the rotations-by-dimensions procecture for orientation differences in different planes.

4. Comparing the results of these two sets of studies is complicated because the two kinds of stimuli differ in several ways: the body is more familiar, has familiar spatial transformations associated with it, and has parts that may be spatially transformed relative to the whole (Parsons, 1987b). 


\section{APPENDIX \\ Spatial Transformation Procedures}

This appendix describes briefly the three spatial transformation procedures considered here (for a more formal treatment, see Parsons, 1987a). Each procedure requires a description of stimulus orientation in direction cosines (or their equivalents). Each procedure is a representative variant of a basic type of approach, of which there are many others.

The rotations-by-dimensions procedure rotates an object through (up to three) Euler angles. Euler angles are commonly used in the physical sciences and engineering to describe objects at different orientations (cf. Goldstein, 1950, p. 107ff.). The degrees of rotation shown in Table 3 are the total degrees of rotation.

The spin-precession procedure discussed here spins the object about its major principal axis. It simultaneously rotates (precesses) it about the axis that is the cross-product of the vectors representing the initial and final orientations of the object's major principal axis. For simplicity, I assume homogeneous motion, so that spin and precession rotations occur at coordinated rates proportional to amount of rotation about each axis neces- sary to correct for the overall orientation difference. The precession angle is that between vectors representing initial and final orientations of the object's major principal axis. The spin angle is found by determining the amount of rotation necessary to align its minor and mean axes once it has rotated through its precession angle. The resulting spatial transformation is a rotation about an instantaneously changing axis. The total angle of rotation about this axis is equal to the precession angle plus the square root of the quantity 1 plus the square of the ratio of spin to precession angles. The expression for the total angle is derived by describing the angular velocity about the spin and precession axes as vectors, and computing the integral of the total length of the vectors for the extent of the precession angle (Parsons, 1987a). In principle, the spin can be about any axis, not just principal axes.

The shortest-path procedure finds the axis (unique for each OD), about which the body can be rotated by an angle absolutely minimizing the degrees of rotation (cf. Parsons, 1987a).

(Manuscript received August 1, 1986; revision accepted for publication February 10, 1987.) 